\title{
Suggestion of a New Brain Reaction Index for the EEG Signal Identification and Analysis
}

\author{
Jungeun $\mathrm{Lim}^{1}$, Bohyeok $\mathrm{Seo}^{2}$ and Soonyong Chun* \\ ${ }^{1}$ School of Electrical Engineering and Computer Science, \\ The Graduate School, Kyungpook National University, Daegu, Korea \\ ${ }^{2}$ School of Electrical Engineering and Computer Science, Kyungpook National \\ University, Daegu, Korea \\ *Electronic Guidance and Control Department, Dongyang University, \\ Yeongju city, Korea \\ euny1122@gmail.com,bhsuh@knu.ac.kr, \\ control@dyu.ac.kr
}

\begin{abstract}
Brain signals are interpreted in various measures. This paper discusses on a research of suggestion or the new brain reaction index during mental calculation from the EEG signals. Measured brain waves were defined as being divided into brain reaction indexes of time and frequency domains for interpreting. 10 men and women were selected as subjects and they solved mathematical questions to find correlation between brain activities and mental calculation. Skewness, kurtosis, BRI and IPSF was applied to the signals in order to extract the EEG parameters. Experimental results indicated that this feature can represent the activity of the left right brain and concentration during mental calculation.
\end{abstract}

Keywords: Brain reaction index, EEG, mental calculation, skewness, kurtosis, Brain Responding Index, Integration of power spectrum Factor

\section{Introduction}

To perform mental calculation for human, brain function operates to deduct results based on logical reasoning as well as to cognize arithmetic regulation. Therefore the studies about measurement and interpretation of brain reaction related to mental calculation can be applied to various branches. These realms are involved not only in cognitive neuroscience researches but also in developmental psychology and evolutional psychology researches, and many studies intended for partial of brain-damaged patients have been progressed.

Even though there are studies about observing changes of blood flow rate utilizing image equipment such as FMRI, brain wave used researches are actively progressed in financial and convenience terms as yet. Diverse ways for measuring brain wave of mental calculation and extracting information of special feature gone through preconditioning process of measured signals are being devised. Signals can be classified by ways of measuring to invasive and noninvasive ways, but $78 \%$ of total theses registered on SCI related to BCI by 2007 are about noninvasive way [1], and studies utilizing noninvasive way electroencephalogram(EEG) among 57 study results submitted to BCI Award 2010 carried out by G.tek are constituting $75.4 \%$ of them [2]. This effect shows that presently EEG based researches are the most active

* Corresponding Author 
ones globally. That is because it is inexpensive and small compared to equipment such as MRI or FMRI with high temporal resolution, although it has demerits of spatial resolution with low EEG. [3]

Brain signals measured during performance of mental calculation are interpreted in various measures using power value or slope of time domain signal, power value of frequency domain(Power Spectral Density: PSD), AR(Auto Regressive) parameter, or variables of coherence, phase synchronization, and phase locking value, or utilizing ERD/ERS or wavelet interpretation.

In this thesis, measured brain waves were defined as being divided into brain reaction indexes of time and frequency domains for interpreting brain reactions during performance of mental calculation. Stochastic feature interpretation measures of skewness and kurtosis were employed on time domain, and brain responding index and integration of power spectrum factor utilizing frequency characteristic of changed brain waves during performance of mental calculation were defined at once.

\section{Interpretation of established EEG signal}

Although EEG signal was interpreted through visual decoding of human in the past, it requires a lot of time and endeavor and has no consistency and objectivity on signal analysis [4].

Therefore, power spectrum analysis classifying from frequency is commonly utilized when observing EEG. Sometimes these distributions of power spectrum are employed with increased number of electrodes of measuring equipment for observing large areas of EEG or for most related portions of research purpose by attaching electrodes on them.

In experimentations concerned with concentration, brain waves are extracted from the frontal lobe part related to recognition ability. General form of EEG utilized for established manner of feature extraction of EEG is as in the following Figure 1.

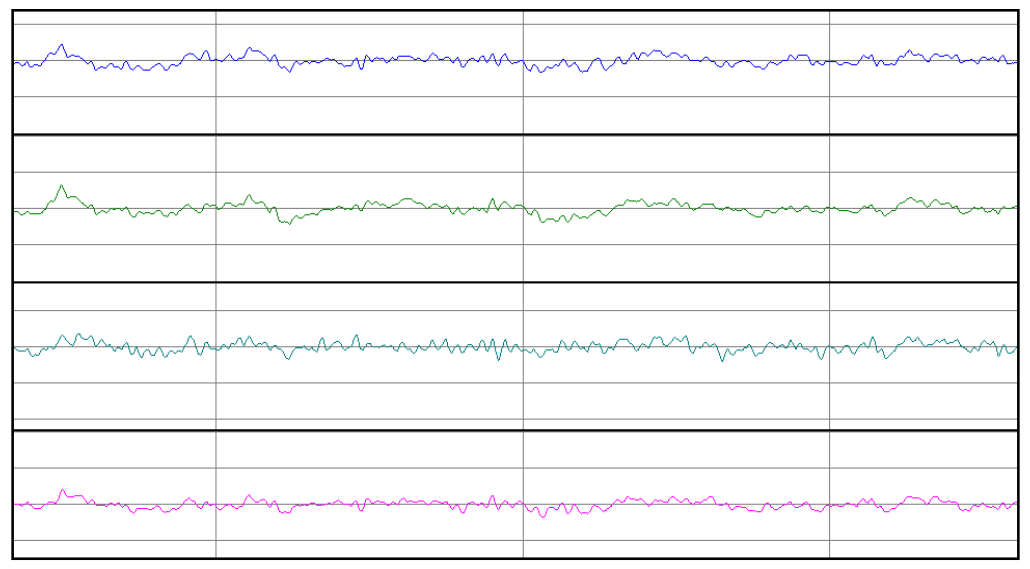

Figure 1. The Reference Signals based on Time Domain

The vertical axis represents amplitude, the horizontal axis represents time. Convert brain waves, measured as a form of figure 1, to frequency domain and compute power spectrums with each frequency domain. Then compare each value or substitute them to specific equation. Equation (1) is generally utilized concentration index formula at present. This is presented as index showing degree of concentration in concentration related experimentations. 


$$
E E G=\frac{S M R+\operatorname{Mid} \beta}{\theta}
$$

In addition to this, there is also analysis method like P300, taking an average of EEG measured at summit attained after about $300 \mathrm{~ms}$ based on presented time of stimulation followed with producing of certain irritation. However, measured data on time domain should be computed with additional algorithm for interpreting on frequency domain. It would be more effective to real-time application than now if computational complexity for processing of EEG can be diminished.

\section{Suggestion of Brain Reaction Index}

Neurons of the frontal lobe taking charge of cognitive management are vitalized when brain starts to perform logical reasoning or mental calculation. Increased regional blood flow rate around them at this moment is much more than increase quantity of oxygen consumption from neurons. Increasing blood flow rate can be perceived with image processors such as FMRI. Although how brain forms action potential through stimulating has not precisely clarified, it is judged to that voltage is formed by $\mathrm{Na}+$ ion penetrating inside and outside of cell, and when blood flow rate increases, forming of $\mathrm{Na}+$ and $\mathrm{K}$ - ions creating action potential from membrane potential gets active, and thus feature of brain wave is changing.

Even though dimension of action potential has no connection with intensity of certain stimulation, repetition rate of action potential increases when intensity of stimulation gets bigger. Interpretation of probability was imposed with perspective of having differences between dimensions of measured EEG from increasing repetition rate of action potential.

Gathered brain wave data were conducted as stochastic variable X. Average is primary moment value and defined with "(2)". It is also called as expected value, and means value dividing aggregate of total signal values with total number of signal data.

$$
\bar{x}=\frac{1}{N} \sum_{i=1}^{N} x_{i}, \quad\left(x_{1}, x_{2}, \cdots, x_{N}\right)
$$

Dispersion defined as secondary moment value is a value defining distant degree from average in probability distribution graph, and is equate to "(3)". Standard deviation is form of having square root on dispersion and defined as "(4)".

$$
\begin{gathered}
\operatorname{Var}\left(x_{1}, x_{2}, \cdots, x_{N}\right)=\frac{1}{N-1} \sum_{i=1}^{N}\left(x_{i}-\bar{x}\right)^{2} \\
\sigma=\sqrt{\operatorname{Var}\left(x_{1}, x_{2}, \cdots, x_{N}\right)}
\end{gathered}
$$

Width of probability distribution graph becomes narrow when standard deviation is small, and this stands for centered data on average, In other words, data values are mostly analogous to average when standard deviation is small, and difference of values is not big.

Furthermore, skewness and kurtosis were computed as criterion for interpretation of stochastic feature of brain waves. Skewness defined as third moment value of signal is a value presenting asymmetrical degree of probability distribution graph and equate to "(5)".

$$
\text { skewness }=\frac{1}{N} \sum_{i=1}^{N}\left\lceil\frac{x_{i}-\bar{x}}{\sigma}\right\rceil^{3}
$$


Signifies that shape of peak in probability distribution is being described to form of generally leaning toward positive sense, if skewness has positive value, and drawn heeling over negative sense, if it has negative value. This can represent the form of probability distribution from data, based on normal distribution

Kurtosis defined as fourth moment value of signal is a value presenting sharpness degree of probability distribution graph and equate to "(6)". It signifies that form of probability distribution graph is sharper than normal distribution if it has positive value and flatness if it has negative value [5].

$$
\text { kurtosis }=\frac{1}{N} \sum_{i=1}^{N}\left\lfloor\left[\frac{x_{i}-\bar{x}}{\sigma}\right]^{4}-3\right.
$$

Existing defined calculation formula of concentration index in frequency domain was defined with new brain reaction index based on experimentation.

BRI (Brain wave responding index) in frequency domain was defined as "(7)" with average and deviation of relative power spectrum from each frequency range.

$$
\begin{aligned}
& \Delta\left|f_{\theta}\right|_{\text {ref }}=\left|f_{\theta}\right|_{\text {ref }}-\left|f_{\theta}\right| \\
& \Delta\left|f_{\alpha}\right|_{\text {ref }}=\left|f_{\alpha}\right|_{\text {ref }}-\left|f_{\alpha}\right| \\
& \Delta\left|f_{\beta}\right|_{\text {ref }}=\left|f_{\beta}\right|_{\text {ref }}-\left|f_{\beta}\right| \\
& B R I=\frac{\Delta\left|f_{\theta}\right|}{\Delta\left|f_{\alpha}\right|+\Delta\left|f_{\text {mid , high } \beta}\right|}
\end{aligned}
$$

Figure 2. shows IPSF(integration of powerspectrum function) of mental calculation in frequency domain. The reference condition were compared as well.

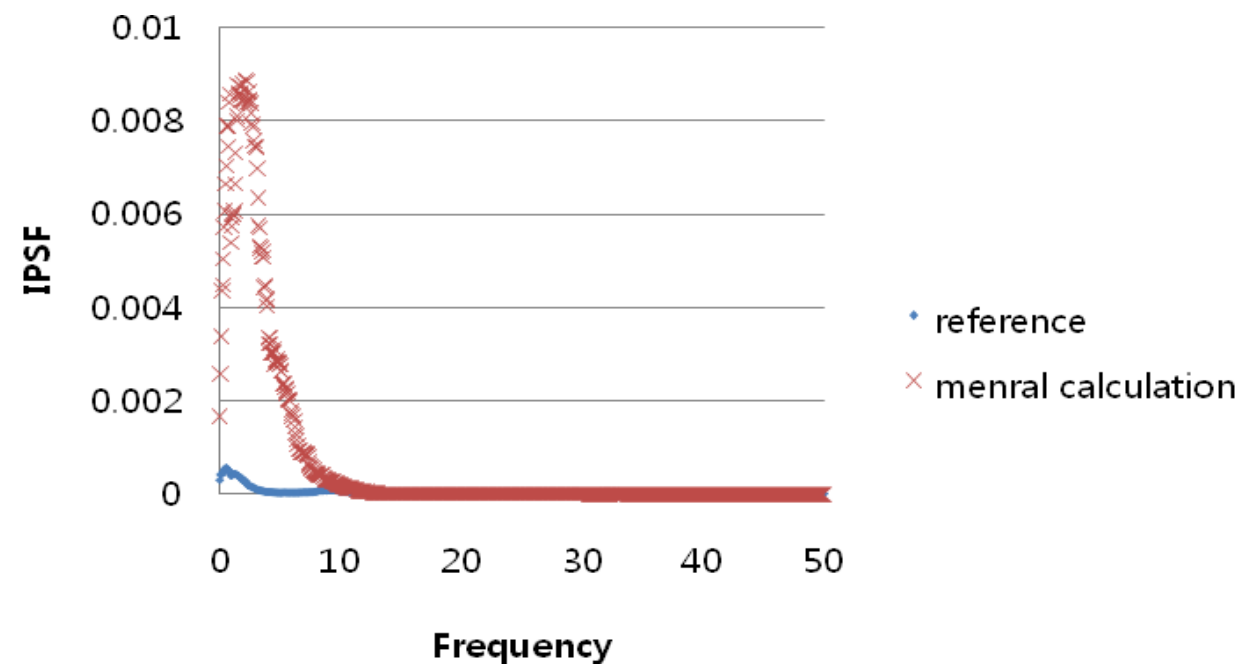

Figure 2. The Reference and Mental Calculation in Frequency Domain 


\section{Material and Methods}

\subsection{Subjects}

This research was imposed conducted with total 10 people of 5 men and women in 20 to 28 years old. Subjects were having no physical and psychological disorders, no coffee, alcohol, and cigarette, and sufficient explanation of experimentation in advance.

\subsection{Experimental Task and Acquisitions}

As EEG was gathered using mp150 of biopac System Company, and employed electrode cab implying international 10-20 electrode system. Sampling rate is $256 \mathrm{~Hz}$ and used both earlobe as reference and ground. Noises from $60 \mathrm{~Hz}$ power-line were eliminated with notch filter. Experimentation was progressed in the room with darkroom structure shutting out extraneous noise and light and maintaining constant temperature and humidity. Subjects are engaged in experimentation with seated condition. Experimenters were participated in followed by sufficient explanation about experimentation in advance. $\mathrm{Fp} 1$ and $\mathrm{Fp} 2$ of prefrontal part, which is a central role of brain function with learning behavior, were measured for brain wave [6].

Criteria brain waves were measured for 60 seconds with comfortable position. Then performed mathematical section questions in limited time for judging mental calculation of subjects, and measured brain wave at this time. Task execution was progressed as a form of calculating addition and subtraction, among the four fundamental arithmetic operations, of double figures maximum and filling in examination paper, and brain waves to 55 questions were measured for 90 seconds. Experimentation was progressed two times as one set of criteria brain wave before performance of task execution and the other set of brain wave during performance of task execution [7].

\section{Experimental Results}

Interpretation value of 10 people with brain reaction index in time and frequency domain during performance of mental calculation were compared on Table 1, considering brain waves measured in comfortable status with eyes closed as criteria condition.

Table 1. Analysis for EEG

\begin{tabular}{ccccc}
\hline \hline reference & Skewness & Kurtosis & BRI & IPSF \\
\hline \hline Min & -1.09384 & 0.21915 & -1.58384 & 0.00315 \\
Max & 0.42851 & 14.2696 & 3.53317 & 0.01202 \\
Mean & -0.05223 & 4.11535 & 0.70253 & 0.00645 \\
Variance & 0.18283 & 24.28629 & 2.1335 & 0.00001 \\
Deviation & 0.42758 & 4.92811 & 1.46065 & 0.00248 \\
\hline \hline $\begin{array}{c}\text { Mental } \\
\text { calculation }\end{array}$ & Skewness & Kurtosis & BRI & IPSF \\
\hline \hline
\end{tabular}




\begin{tabular}{ccccc}
\hline \hline reference & Skewness & Kurtosis & BRI & IPSF \\
\hline \hline Min & -0.42166 & 1.68257 & 1.26196 & 0.04345 \\
Max & 1.42178 & 12.91537 & 31.34982 & 0.24508 \\
Mean & 0.42138 & 6.23102 & 1.30476 & 0.09478 \\
Variance & 0.23858 & 14.90132 & 0.00074 & 0.0035 \\
Deviation & 0.48845 & 3.86022 & 0.02717 & 0.05912 \\
\hline
\end{tabular}

As you can see in the table, probability elements in time domain with criteria condition with nonperformance of mental calculation were -0.05223 as skweness, 4.11535 as kurtosis, 0.70253 as frequency domain value in average, and 0.00645 as IPSF. However, they got bigger as skewness to 0.42138 and kurtosis to 6.23102 when performing mental calculation. BRI in frequency domain got bigger as 1.30476 in averages, about 2 times bigger than criteria condition, and IPSF got larger about 10 times more as 0.09478. Individual factor values of each condition are presented on Figure 3. The Skewness values in time domain are having positive values during performance of mental calculation in principle, because of increasing repetition rate of action potential with growth number of ions from swelling blood flow rate during performance of mental calculation of brain. Kurtosis was also growing from repetitive formation of specific potential through increasing of changes in brain blood flow rate for particular stimulation when performing mental calculation. Although calculation formula BRI in frequency domain had big deviations for each individual, it converged to about 1.3 during performance of mental calculation. IPSF increases largely and this presents that brain is actively operating than in general state.

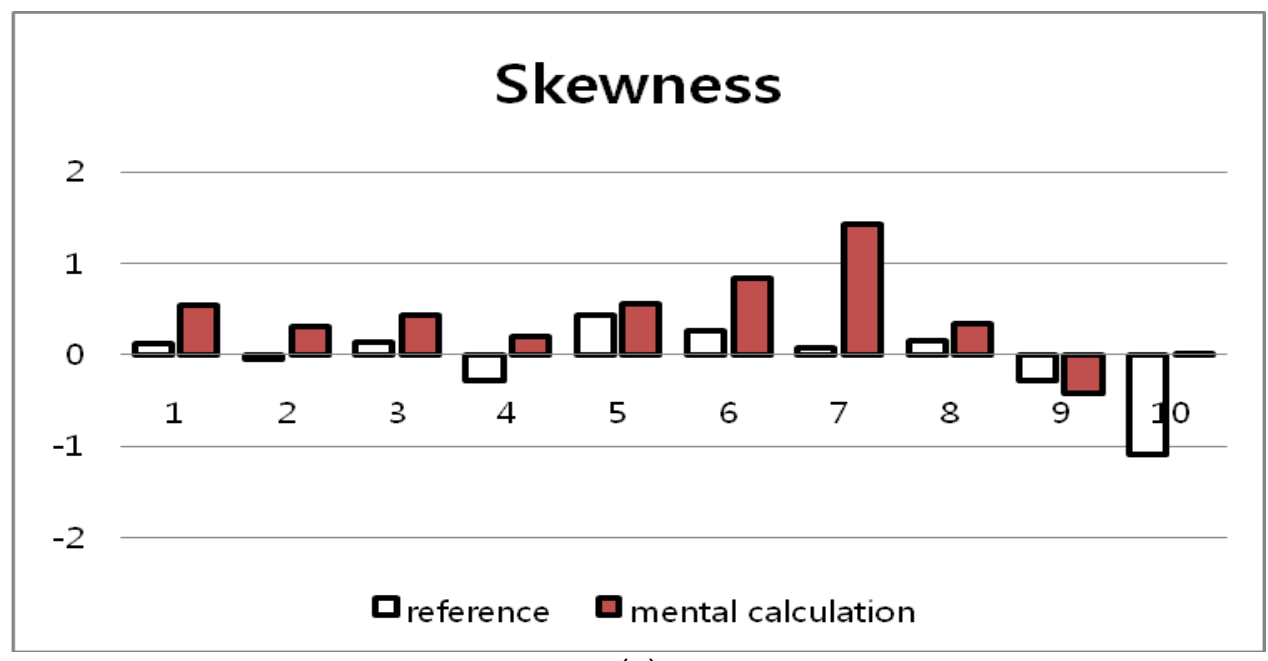

(a) 


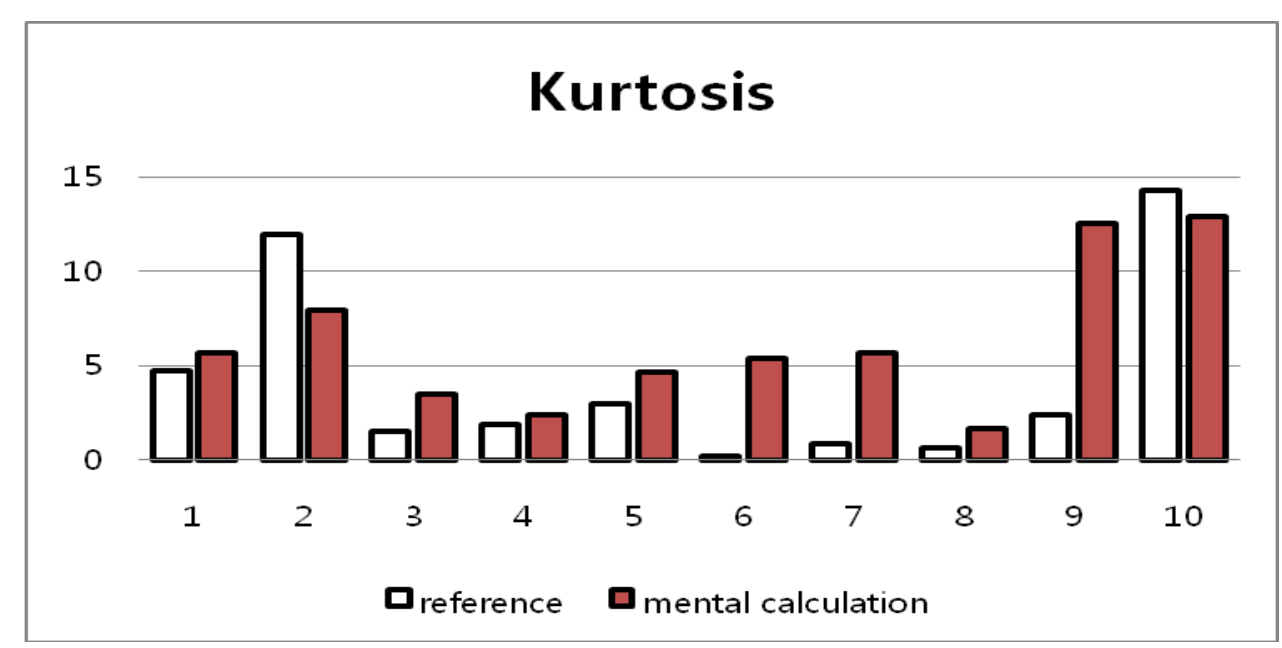

(b)

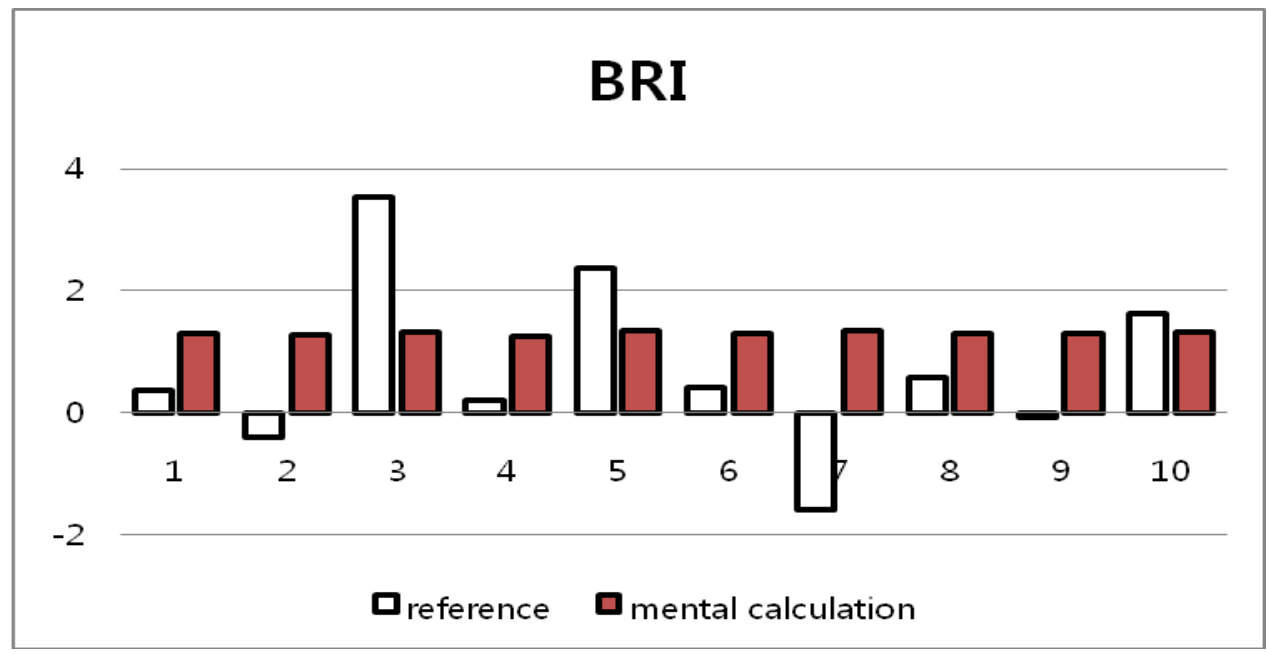

(c)

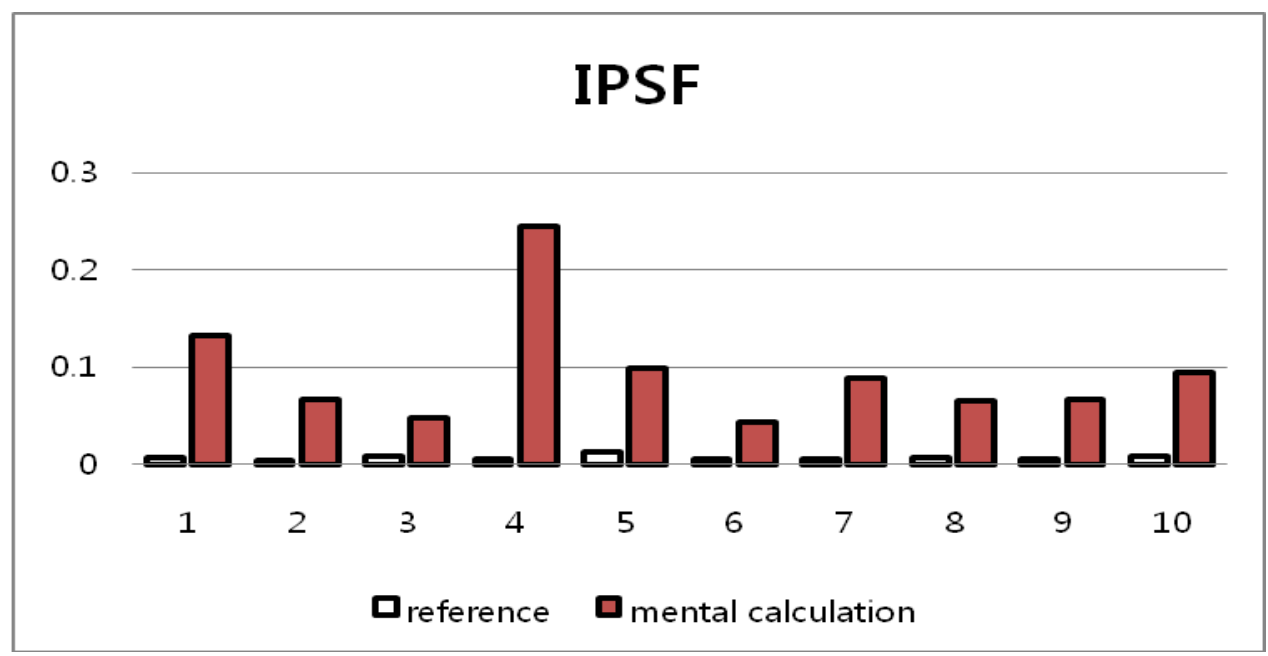

(d)

Figure 3. Brain Reaction Index 


\section{Conclusion}

In this research, new brain reaction index in frequency and time domain was proposed with performing mental calculation and measuring brain wave. Brain wave experiment was progressed in regular times for performing mental calculation, using double figures with addition and subtraction among the four fundamental arithmetic operations. Interpretation in time domain was applied to stochastic techniques of skewness and kurtosis and compared with thoses values, and it proposed new brain responding index.

When performing mental calculation comparing with brain wave of criteria condition, skewness and kurtosis values were increasing toward positive sense. This signifies that repetition rate of action potential increases from biological brain reaction for specific stimulation of calculating, and thus distributions of data peak are not having symmetry but having focused on positive sense. Additionally increasing of kurtosis value stands for sharpness value than normal distribution. This can be interpreted as frequent emerging of specific frequency than general status during performance of mental calculation. Also alpha wave of $8 \mathrm{~Hz}$ to $13 \mathrm{~Hz}$ was decreased in frequency interpretation, and theta wave of $4 \mathrm{~Hz}$ to $8 \mathrm{~Hz}$ was increased. Proportion of $15 \mathrm{~Hz}$ to $30 \mathrm{~Hz}$ in beta wave domain was irregular but showing decrease trend. Therefore, BRI was defined utilizing power spectrum value in those three rages. Average BRI of 10 people from experimentation objects were about 1.3 during performance of mental calculation. IPSF value is also increase. It means mental calculation rev up action potential. These results are the average of two measurement points. If this value is calculated at each point, the value of left brain and right brain are slightly different. So, BRI and IPSF calculated in the frequency domain especially can be useful in the assessment of left and right brain activation. Skewness and kurtosis are useful factor for evaluating of concentration.

Proposing of new brain wave index made established brain wave analysis and other forms of brain wave analyzing possible. It presents possibility of applying not only on brain reaction research of mental calculation but also interpretation of other forms of brain activities.

\section{References}

[1] C.-H. Lim, "Introduction of brain-computer interface technology based on brain wave", J. Biomed. Eng., vol. 31, (2010), pp. 1-13.

[2] Reza Fazed, "Recent Advances in brain-computer Interface systems: 10 state of the Art in BCI Research: BCI Award 2010”, In Tech, (2011).

[3] B.k. Min, "Spectral analysis of brain oscillatory activity", Korean Journal of Cognitive Science 2009, vol. 20, No. 2, (2009), pp. 155-181.

[4] J.Lim, B. Seo and S. Chun, "Study on EEG feature extraction under LED color exposure to enhance the concentration", Advanced Engineering Forum, vol. 2-3, (2012), pp.261-265.

[5] M.-S. Chun, Mathematical Statistics, Freedom Academy, (2008)

[6] A.E.Lawson, "The role of the prefrontal lobes in scientific reasoning", The Korea Association for Science Education, vol 17, no. 4, (1997), pp.525-540.

[7] J. Lim, U. Ji, B. Seo, S. Chun, "Measurements of color effects on the brain activity for eye-attention", ICROS, (2012). 


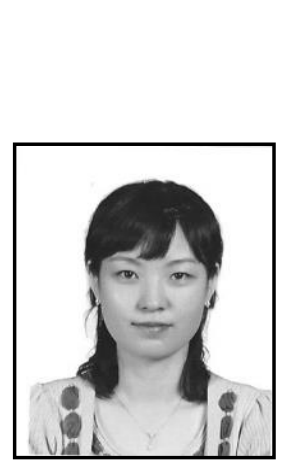

\section{Authors}

Jungeun Lim received the B.S. and M.S. degree in Electrical Engineering and Computer Science from Kyungpook National University, Daegu, Korea in 2005 and 2007. She is currently working toward her $\mathrm{Ph} \mathrm{D}$. degree in Electrical Engineering and Computer Science at Kyungpook National University, Daegu, Korea. Her recent interests focus on Biomedical Engineering.

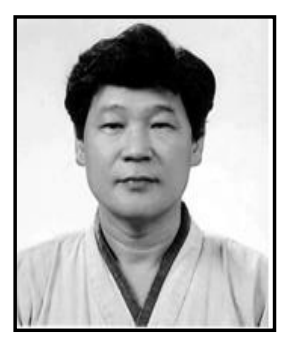

Bohyeok Seo received the B.S., M. S. and the Ph.D. degrees in Electrical Engineering from Seoul National University, Seoul, Korea, in 1975, 1980 and 1983 respectively. Since 1983 he has been with the school of Electrical Engineering and Computer Science, Kyungpook National University, Daegu, Korea, where he is currently a professor. His recent interests focus on control systems.

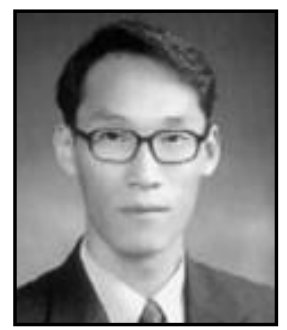

Soonyong Chun received the B.S., M. S. and the Ph.D. degrees in Electrical Engineering from Kyungpook National University, Daegu, Korea, in 1986, 1991 and 1995 respectively. Since 1996 he has been with Electronic Guidance and Control Department, Dongyang University, Yeongju city, Korea, where he is currently a professor. $\mathrm{He}$ is also currently a registration director of Integrative Medicine Promotion \& Research Institute. Moreover, he is a Chief of dept. of cooperation/registration director at Industrial Control Technology Institute and President of National Defense Technology Institute. His recent interests focus on control theory application, measurement system design for human body and national defense technology. 
International Journal of Bio-Science and Bio-Technology Vol.6, No.4 (2014) 